\title{
Assessment of set-up errors in the radiotherapy of patients with head and neck cancer: standard vs. individual head support
}

\author{
Sabina Androjna ${ }^{1}$, Valerija Zager Marcius ${ }^{1,2}$, Primoz Peterlin ${ }^{1}$, Primoz Strojan ${ }^{1,3}$ \\ ${ }^{1}$ Department of Radiotherapy, Institute of Oncology Ljubljana, Ljubljana, Slovenia \\ ${ }^{2}$ Faculty of Health Sciences, University of Ljubljana, Ljubljana, Slovenia \\ ${ }^{3}$ Faculty of Medicine, University of Ljubljana, Ljubljana, Slovenia \\ Radiol Oncol 2020; 54(3): 364-370.
}

Received 17 February 2020

Accepted 3 May 2020

Correspondence to: Prof. Primož Strojan, M.D., Ph.D., Institute of Oncology Ljubljana, Zaloška 2, SI-1000 Ljubljana, Slovenia. E-mail: pstrojan@onko-i.si

Disclosure: No potential conflicts of interest were disclosed.

Background. The aim of the study was to (a) compare the accuracy of two different immobilization strategies for patients with head and neck tumors, and (b) compare the set-up errors on treatment units with different portal imaging systems.

Patients and methods. Variations in the position of the isocenter (IC) relative to the reference point determined on the computed tomography simulator were measured in a vertical (anterior-posterior), longitudinal (superior-inferior), and lateral (medial-lateral) direction in 120 head and neck cancer patients irradiated with curative intent. Depending on the treatment unit (unit A - 2D/2D image previews; unit B - 2D image previews) and the time of irradiation, patients were divided into 6 groups of 20 patients. In patients irradiated in 2014, standard head supports were used (groups 1 and 2), whereas in those treated in 2015 and 2017 (groups 3-6) individual head supports were employed. The clinicalto-planning target volume safety margin was calculated according to the formula proposed by Van Herk.

Results. In total, 2,454 portal images and 3,681 set-up errors were analysed. Implementation of individual head supports in 2015 resulted in a statistically significant reduction in the average inter-fraction displacement in the vertical direction and in decreased number of IC displacements in the vertical and longitudinal direction (applies to both treatment units). The largest reduction of the safety margin was calculated in the longitudinal direction and the safety margins were larger for unit $B$ than for unit $A$.

Conclusions. The use of individual head supports and a more advanced imaging system were found to increase set-up precision.

Key words: head and neck radiotherapy; immobilization; head support; set-up errors

\section{Introduction}

The basic tools for the immobilization of patients with head and neck $(\mathrm{HN})$ tumors during radiotherapy are thermoplastic masks with a 5-point pinning system and supporting system for the head. These immobilization aids largely, but not completely, prevent major shifts during irradiation. Due to its regular use, the head support can shrink and deform over time, which leads to deviations in the position of the $\mathrm{HN}$ compared to the reference position determined on the computed tomography (CT) simulator (Figure 1). To overcome this problem, patient-specific head supports were introduced (i.e., customized head support that are moulded to the patient's anatomy), which proved to effectively reduce systematic and random errors. ${ }^{1-4}$

At the Institute of Oncology Ljubljana, the majority of $\mathrm{HN}$ cancer patients are irradiated on two similar treatment units with slightly different imaging capabilities. Commercially available head 
supports have been used since the 1990s (CIVCO, Coralville, Iowa, USA), whereas patient-specific head supports have never been introduced in routine practice. Until 2015, all patients irradiated on a particular treatment unit shared the same set of head supports (i.e., standard head support). In order to reduce the set-up error, this policy was changed in 2015 and it was ensured that the same head support was used for a given patient from the CT simulator throughout the irradiation course (i.e., individual head support). Regular quality checks of head supports were performed: the difference between the heights of used and unused supports was not allowed to exceed $3 \mathrm{~mm}$.

In the present study, two hypotheses were tested: (1) Deviations recorded by portal imaging system will be smaller in patients using individual head supports compared to those with standard ones; (2) The treatment unit with a more advanced portal imaging system will allow for a more accurate positioning of patients.

\section{Patients and methods}

Between January 2014 and October 2017, 120 HN cancer patients irradiated with curative intent were included in this retrospective non-interventional study. Patients were irradiated on either of the two low-energy linear accelerators equipped with MV imaging systems: unit A - Unique Performance Edition; and unit B - Clinac DBX (both: Varian Medical Systems, Palo Alto, California, USA). Depending on the treatment unit (A or B), the time of irradiation, and the type of head support used, patients were divided into 6 groups of 20 patients. Because individual head supports were introduced into routine practice in 2015, the consistency of results related to their use over time was verified in two time periods (2015 and 2017) and, consequently, in two independent groups of patients:

- Group 1 - linear accelerator A, 2014

(standard head support)

- Group 2 - linear accelerator B, 2014

(standard head support)

- Group 3 - linear accelerator A, 2015

(individual head support)

- Group 4 - linear accelerator B, 2015

(individual head support)

- Group 5 - linear accelerator A, 2017

(individual head support)

- Group 6 - linear accelerator B, 2017

(individual head support)
Variations in the position of the isocenter (IC) relative to the reference point determined on the CT simulator (i.e., set-up errors) were measured in a vertical (anterior-posterior), longitudinal (superior-inferior), and lateral (medial-lateral) direction.

\section{Simulation procedure}

At the CT simulator, the most appropriate head support was selected from the commercially available set of items of various heights and contours, offering a comprehensive range of neck angulations (CIVCO, Coralville, Iowa, USA), according to the curvature of patient's neck and occiput. The Kneefix $^{\mathrm{TM}}$ and the Armaflex ${ }^{\mathrm{TM}}$ cushion were placed under the knees and the back and pelvis, respectively, and the head was additionally fixed with a thermoplastic 5-point Posicast ${ }^{\circledR}$ mask (all: CIVCO, Coralville, Iowa, USA). Radio-opaque markers (Beekley Medical, Bristol, Connecticut, USA) were used for three-point marking of the IC origin. CT scanning from $2 \mathrm{~cm}$ above the top of the head to the tracheal bifurcation (slice thickness: $2 \mathrm{~mm}$ ) was accomplished using an intravenous administration of iodine contrast medium by power injector, followed by tattooing the thoracic skin for the central alignment of the patient.

\section{Geometric verification}

Patients on the treatment units were pre-positioned into the IC, based on the room lasers before the set up imaging. Portal images were taken according to the Extended No Action Level (eNAL) protocol. ${ }^{5}$ The PortalVision computer program with

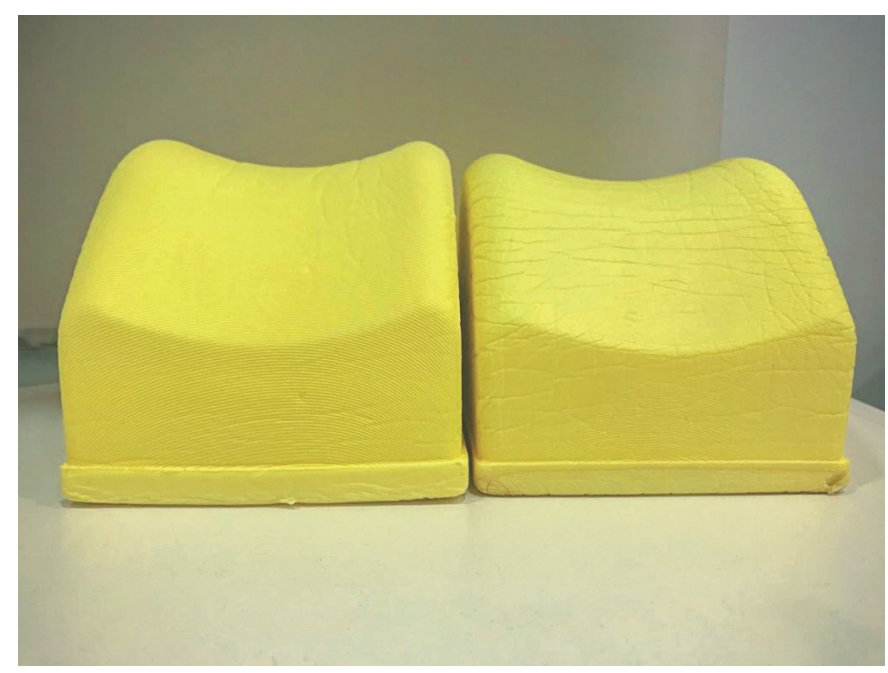

FIGURE 1. The example of shrinkage (right) of the head support. 


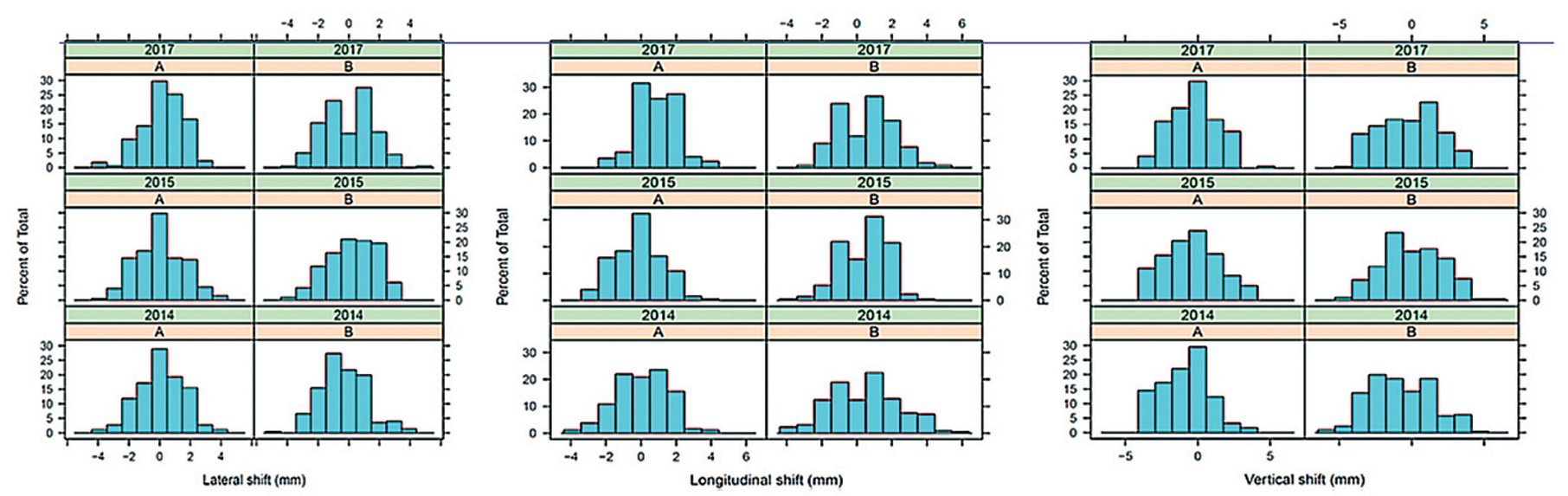

FIGURE 2. Inter-fraction displacements by axis and period.

the AutoMatching registration procedure (Varian Medical Systems, Palo Alto, California, USA) was used to calculate the size and direction of the displacement. Electronic portal images acquired with gantry at $0^{\circ}$ (anteroposterior projection) and $90^{\circ}$ (or $270^{\circ}$, lateral projection) were compared with digitally reconstructed radiographs (DRRs). Portal images were obtained using the Varian's EPID PortalVision using an amorphous silicon plane detector aS1000 (resolution of $1024 \times 768$ pixels, unit A) or aS500 (resolution $512 \times 384$ pixels, unit B). Whereas unit A allows simultaneous patient position alignment in all three directions using 2D/2D image matching, unit $\mathrm{B}$ requires the radiographers to combine the position corrections obtained from two separate orthogonal 2D image matchings.

\section{Statistical analysis}

The study protocol was approved by the Protocol Review Board of the Institute of Oncology Ljubljana on April 4, 2017.

Testing set-up error distributions for normality was done using the Shapiro-Wilk test. As ShapiroWilk test did not support the normality hypothesis in any of the distribution, non-parametric MannWhitney U-test (two-sample rank-sum test) was employed for comparing median values in distributions instead of two-sided Student's t-test. For the same reason, non-parametric modified Levene's test (using median instead of mean) was used to test the equality of variances in set-up error distributions. Statistical calculations were performed using the GNU R statistical program. ${ }^{7}$ The sample size of 350 measurements per time period was calculated with the $\mathrm{G}^{*}$ Power software, considering $a=0.05, b=0.8$ and the effect size of 0.267 , which was calculated on the basis of averages and standard deviations of similar studies., ${ }^{1,2,6,8}$ For comparison with published studies, the clinical target volume (CTV) - planning target volume (PTV) safety margin was calculated according to the formula proposed by Van Herk. ${ }^{9}$ The differences at $\mathrm{p}$ $<0.05$ were considered statistically significant.

\section{Results}

In total, 2454 portal images and 3681 set-up errors were analysed: 828,832 , and 794 portal images obtained in 2014, 2015, and 2017, respectively. We first analysed the data sets for the presence of large setup errors. The proportion of displacements smaller than $3 \mathrm{~mm}$ and smaller than $5 \mathrm{~mm}$ were $85 \%$ and $99.1 \%$, respectively in the 2014 set, when standard head supports were employed. The introduction of individual head supports in 2015 increased these figures to $89 \%$ and $99.6 \%$, respectively, and in a most recent data set from 2017 they were further increased to $90 \%$ and $99.6 \%$, respectively.

Inter-fraction set-up errors registered in units $\mathrm{A}$ and $\mathrm{B}$ at different periods are shown in Table 1 and Figure 2. The difference in distribution of interfraction displacements were tested using MannWhitney U-test. In four cases, the test showed that the distribution obtained in one year differ significantly $(p<0.05)$ from those obtained in the other two years. For unit A, set-up error distributions in the vertical direction obtained in 2014 and in the longitudinal direction obtained in 2017 differ from the other two years. For unit B, set-up error distributions in the vertical direction in the year 2014 and in the lateral direction in the year 2014 differ from the other two years. Comparing the vertical shift distribution for the unit A in the years 2014 and 2015 shows significant difference (Mann-Whitney U = 
TABLE 1. Inter-fraction displacements recorded on units $A$ and $B$ at different periods

\begin{tabular}{|c|c|c|c|c|c|c|c|c|c|}
\hline & \multicolumn{3}{|c|}{$\begin{array}{c}2014 \\
\text { Standard head support }\end{array}$} & \multicolumn{3}{|c|}{$\begin{array}{c}2015 \\
\text { Individual support }\end{array}$} & \multicolumn{3}{|c|}{$\begin{array}{c}2017 \\
\text { Individual support }\end{array}$} \\
\hline \multicolumn{10}{|l|}{ UNIT A } \\
\hline Average displacement - $M$ [mm] & -0.86 & 0.05 & 0.05 & $-0.47^{*}$ & $-0.24^{*}$ & -0.05 & $-0.16^{*}$ & 0.88 & 0.18 \\
\hline Systematic error $-\Sigma[\mathrm{mm}]$ & 0.66 & 1.04 & 0.88 & 0.91 & 0.79 & 0.95 & 0.82 & 0.59 & 0.83 \\
\hline Random error - $\sigma[\mathrm{mm}]$ & 1.49 & 1.21 & 1.37 & 1.56 & 1.28 & 1.38 & 1.28 & 1.14 & 1.2 \\
\hline Average displacement - $M$ [mm] & -0.60 & 0.46 & -0.43 & $-0.02 *$ & 0.51 & 0.25 & $-0.18^{*}$ & 0.46 & -0.02 \\
\hline Systematic error $-\Sigma[\mathrm{mm}]$ & 1.09 & 0.80 & 0.82 & 0.92 & 0.74 & 0.94 & 0.93 & 0.69 & 0.88 \\
\hline Random error - $\sigma[\mathrm{mm}]$ & 1.77 & 1.83 & 1.47 & 1.77 & 1.22 & 1.41 & 1.71 & 1.56 & 1.44 \\
\hline
\end{tabular}

LAT = lateral (medial-lateral); LNG = longitudinal (superior-inferior); VRT = vertical (anterior-posterior)

${ }^{*} p<0.05$ (2014 vs. 2015 or 2014 vs. 2017)

16293, n1 = 187, n2 = 201, p=0.02, Hodges-Lehmann estimator $(\mathrm{HL} \Delta)=-0.000012,95 \%$ confidence interval (CI) is $(-0.999974,-0.000010))$. The difference in distributions is even more pronounced between the sets for the year 2014 and 2017 ( $U=12918.5, n 1=$ $187, \mathrm{n} 2=175, \mathrm{p}<0.001, \mathrm{HL} \Delta=-0.999982,95 \% \mathrm{CI}$ (-0.999989, -0.000059). Comparing the distributions for the years 2015 and 2017 did not show a significant difference. Comparing the longitudinal shift distributions for the unit A also doesn't show a significant difference; however, comparing the distributions for the years 2014 and 2017 does show a significant difference $(\mathrm{U}=11230, \mathrm{n} 1=187, \mathrm{n} 2=$ 175, $\mathrm{p}<0.001$, HL $\Delta=-0.999982,95 \%$ CI (-1.000066, -0.999956)), and so does the comparison for the years 2015 and 2017 ( $\mathrm{U}=10171.5, \mathrm{n} 1=201$, n2 = $175, \mathrm{p}<0.001, \mathrm{HL} \Delta=-1.000006,95 \%$ CI $(-1.000032$, $-0.999955)$ ). Neither comparison for the lateral shifts for the unit A showed significance. Comparing the vertical shift distributions for the unit $\mathrm{B}$ also shows significant difference between the sets for the years 2014 and $2015(\mathrm{U}=19637.5, \mathrm{n} 1=227, \mathrm{n} 2=$
215, $\mathrm{p}<0.001, \mathrm{HL} \Delta=-0.999931,95 \%$ CI $(-1.000049$, $-0.000042))$, as well as between the sets for the years 2014 and $2017(\mathrm{U}=21674, \mathrm{n} 1=227, \mathrm{n} 2=222, \mathrm{p}<0.01$, HL $\Delta=-0.000046$, 95\% CI $(-0.999990,-0.000025))$, while the difference between data sets for the years 2015 and 2017 is not significant. In unit B, none of the differences in the longitudinal shift distribution is considered significant. Comparing the lateral shift distributions shows significance between the data sets for the years 2014 and 2015 (U = 18998, n1 $=227, \mathrm{n} 2=215, \mathrm{p}<0.01, \mathrm{HL} \Delta=-0.999942,95 \% \mathrm{CI}$ $(-0.999980,-0.000042))$ and for the years 2014 and $2017(\mathrm{U}=22030, \mathrm{n} 1=227, \mathrm{n} 2=222, \mathrm{p}<0.02, \mathrm{HL} \Delta$ $=-0.000058,95 \%$ CI $(-0.999953,-0.000040))$, while the distributions of lateral shifts between 2015 and 2017 is not considered significant. Comparing the variances of the distributions (which correspond to the systematic error $\Sigma$ and the random error $\sigma$ combined) only shows significant differences in five cases: in unit A the vertical shift distributions for the years 2015 and 2017 differ significantly (Levene's F $=6.3082, \mathrm{DF}=386, \mathrm{p}<0.02)$, as well as longitudinal

TABLE 2. Number of IC displacements and of gross errors at treatment units in relation to time for both units. In the brackets, the most prevalent direction of applied movements is indicated

\begin{tabular}{|c|c|c|c|c|}
\hline & \multicolumn{3}{|c|}{ Isocenter movements } & \multirow{3}{*}{ Gross error: } \\
\hline & VRT & LNG & LAT & \\
\hline & $\begin{array}{c}\text { Unit A / unit B } \\
\text { (direction) }\end{array}$ & $\begin{array}{l}\text { Unit A / unit B } \\
\text { (direction) }\end{array}$ & $\begin{array}{c}\text { Unit A / unit B } \\
\text { (direction) }\end{array}$ & \\
\hline 2014 - standard head support & $11 / 15(\mathrm{P})$ & $5 / 15$ (1) & $4 / 5$ (L) & $2 / 5$ \\
\hline 2015 - individual head support & $12 / 13(P)$ & $3 / 6$ (I) & $9 / 8(R)$ & $0 / 1$ \\
\hline 2017 - individual head support & $5 / 14(P)$ & $7 / 10$ (l) & $6 / 7(\mathrm{~L})$ & $0 / 1$ \\
\hline
\end{tabular}

$\mathrm{I}=$ inferior; $\mathrm{L}=$ left; $\mathrm{LAT}=$ lateral (medial-lateral); $\mathrm{LNG}=$ longitudinal (superior-inferior); $\mathrm{P}=$ posterior; $\mathrm{R}=$ right; VRT = vertical $($ anterior-posterior) 
TABLE 3. Clinical target volume - planning target volume (CTV-PTV) safety margins for units $A$ and $B$ at different periods (calculated according to van Herk ${ }^{8}$ )

\begin{tabular}{llll}
\hline & \multicolumn{3}{c}{ CTV-PTV safety margin } \\
\cline { 2 - 4 } & VRT & LNG & LAT \\
\hline UNIT A & & & \\
$\mathbf{2 0 1 4}$ - standard head support & 2.7 & 3.4 & 3.2 \\
$\mathbf{2 0 1 5}$ - individual head support & 3.4 & 2.9 & 3.3 \\
$\mathbf{2 0 1 7}$ - individual head support & 2.9 & 2.3 & 2.9 \\
UNIT B & & & \\
$\mathbf{2 0 1 4}$ - standard head support & 4.0 & 3.3 & 3.1 \\
$\mathbf{2 0 1 5}$ - individual head support & 3.5 & 2.7 & 3.3 \\
$\mathbf{2 0 1 7}$ - individual head support & 3.5 & 2.8 & 3.2 \\
\hline
\end{tabular}

LAT = lateral (medial-lateral); LNG = longitudinal (superior-inferior); VRT = vertical (anterior-posterior)

shift distributions for the years 2014 and $2017(\mathrm{~F}=$ 9.2817, $\mathrm{DF}=360, \mathrm{p}<0.01)$. In unit $\mathrm{B}$, all three comparisons of longitudinal shift distributions show significant difference: between the sets for 2014 and $2015(\mathrm{~F}=24.5077, \mathrm{DF}=440, \mathrm{p}<0.001)$, between the sets for 2014 and $2017(\mathrm{~F}=9.1372, \mathrm{DF}=447, \mathrm{p}<$ $0.01)$, and between the sets for 2015 and $2017(\mathrm{~F}=$ $4.5802, \mathrm{DF}=435, \mathrm{p}=0.03)$. In Table 2 , the number of IC displacements for both units together at different periods are presented, as well as the number of recorded gross errors $(>5 \mathrm{~mm})$. Most of the IC shifts were made in the posterior, inferior and in the left direction. With the implementation of individual head supports, their number decreased, except for the lateral direction, and the number of gross errors was also reduced.

The CTV-PTV safety margins calculated from the population set-up errors for units A and B at different periods are shown in Table 3. In unit A, the largest reduction of the safety margin after implementation of individual head supports was calculated in the longitudinal direction (2014 vs. 2015, by 0.6 ; and 2014 vs. 2015, $1.2 \mathrm{~mm}$ ), whereas in the lateral direction, the margin did not change substantially. On the contrary, in the vertical direction the margin increased by $0.7 \mathrm{~mm}$ (2014 vs. 2015) and by $0.2 \mathrm{~mm}$ (2014 vs. 2017). In unit B, a general trend toward a reduction in the safety margins resulted from the employment of individual head supports. In addition, the average reduction of the safety margins was also larger in unit B. The most significant reductions (2014 vs. 2015 and 2017) were observed in the vertical (by 0.4 and $0.5 \mathrm{~mm}$ ) and longitudinal directions (by 0.6 and $0.5 \mathrm{~mm}$ ). In the lateral direction, the size of the safety margin did not increase substantially (by 0.3 and $0.1 \mathrm{~mm}$ ).

\section{Discussion}

In the present study, individual head supports were found to significantly reduce inter-fraction displacements in the vertical direction, specifically in the posterior direction, compared to the standard head supports. Reduction of average displacement in vertical direction recorded between 2014 and 2017 on units A and B was $0.70 \mathrm{~mm}$ and 0.42 $\mathrm{mm}$, respectively. This observation pointed to the shrinkage of material, i.e. polyurethane foam, as a possible reason for the observed displacements due to the prolonged and frequent use of head supports.

Comparing the three periods, the systematic error did not change significantly for either unit. In the vertical direction, the systematic error recorded on unit A increased by an average of $0.15 \mathrm{~mm}$, while on unit B it decreased by approximately the same extent. A negligible increase over the time was observed in the lateral direction, on average by less than $0.1 \mathrm{~mm}$. It seems that the use of head supports and the shrinkage of the material they are made of influenced mainly the rotational set-up errors of the head in the sagittal plane, rather than the head displacements to the left or to the right. ${ }^{1}$ In the longitudinal direction, the systematic error was reduced over time on both treatment units. Similarly, by abolishing the standard head supports, a statistically non-significant decrease in the size of the random error was recorded on unit $\mathrm{A}$ in all three directions. On unit $B$, the random error remained practically unchanged in two directions; in the longitudinal direction, its change was negligible.

Our observations are in line with those of other authors. A reduction of systematic and random errors in all directions was calculated by Van Lin et al. ${ }^{1}$ when customized and the standard head supports were compared. A decrease was most notable in the longitudinal direction and least marked in the lateral direction, which is the pattern comparable to that found in our study. McKernan et al. showed a reduction in setup error by on average $1.3 \mathrm{~mm}$ with the use of customized head supports instead of standard ones. ${ }^{4}$ Similarly, Houweling et al. reported that the use of customized head supports reduced systematic errors by at least $20 \%$ and random errors by at least $25 \%{ }^{2}$ They indicated a decrease in inter-fraction set-up errors by $40 \%$; 
most statistically significant displacements were recorded in the lateral direction. However, in this particular direction we recorded the smallest setup errors. The observed discrepancy could be due to sample characteristics: this was significantly smaller $(\mathrm{n}=22)$ in the study of Houweling et al. than in our study $(n=120)$. Thus, their results are less likely to adequately represent the characteristics of the population. To the contrary, in the study of Howlin et al., the difference in set-up errors between patients with customized and standard head supports was not significant in any direction. ${ }^{6}$

Furthermore, our calculations of the estimated margins from CTV to PTV were also comparable to those reported in the literature. Humphreys et al. used a customized immobilization system: the estimated margins in lateral, longitudinal and vertical directions were $2.9,2.6$ and $3.3 \mathrm{~mm}$, respectively. ${ }^{3}$ The authors used the same formula as we did. ${ }^{9}$ Similarly, Van Lin et al. suggested that with a customized head support and appropriate correction protocol, suitable CTV-PTV margins would be $3 \mathrm{~mm}$ in the vertical and longitudinal directions and $4 \mathrm{~mm}$ in the lateral direction. ${ }^{1}$ However, we observed that the CTV-PTV safety margins were larger for unit B than for unit A, which confirms our second hypothesis that the treatment unit with a more advanced portal imaging system allows for more accurate positioning of patients.

Humphreys et al. ${ }^{3}$ reported $94 \%$ of displacements smaller than $3 \mathrm{~mm}$ and $99 \%$ smaller than $5 \mathrm{~mm}$, which is comparable to the results of this study. In addition, individual head supports reduced the number of IC set-up errors in our patients, particularly in the vertical direction, and also of gross errors by $66 \%$; all but two of the latter were recorded in the posterior direction. In unit $\mathrm{A}$, which was equipped with a more advanced portal imaging system, fewer IC displacements and fewer gross errors were documented than in unit B.

In addition, there were some differences across the study groups recorded in the size of inter-fraction displacements (unit A: longitudinal displacement, 2014 vs. 2017), number of IC displacements (unit A: longitudinal axis, 2014 vs. 2015 vs. 2017), and in the size of CTV-PTV margin (unit A: vertical axis, 2014 vs. 2015 vs. 2017), which are not in line with the expected greater accuracy when using individual head rests. However, these differences are small and, as such, seem to be of questionable importance for day-to-day clinical work. We are aware that there may be more causes for registered set-up errors that may also influence the calculation of the CTV-PTV margin; imprecision in daily set-up and patient movements when lying on the table of treatment unit are just two of the potential sources..$^{10} \mathrm{As}$ measurements within each of the six study groups were made within a relatively short time (i.e. 10-12 weeks) and with constant RTT teams, it can be argued that the results of the group measurements were consistent. Of course, over the 2015-2017 period, there were changes in the composition of RTT teams, which could affect our calculations. Other causative factors for set-up errors would be different technical errors (inaccuracies in the in-room laser calibration or of the imaging IC, procedure of the matching process and its quality) or those originated from the thermoplastic mask itself, changes in the patient anatomy (due to weight loss or volume reduction/swelling of the tumor or specific organs-at-risk), or different physiological processes (swallowing respiration). However, we were able to account for these factors only in the context of a regular quality assurance program; their detailed analysis is beyond the scope of this study. The impact of eventual changes in the departmental protocol used to position patients on irradiation units is negligible, since no significant protocol changes occurred during the study period.

\section{Conclusions}

When compared to standard head supports, the introduction of individual head supports reduced inter-fraction set-up errors in the vertical direction and the number of gross errors; in some directions, also the number of IC displacements and the size of the CTV-PTV safety margin were reduced. A more advanced imaging system with a better spatial resolution contributed to a reduction in the systematic and random errors.

\section{Acknowledgement}

This work was financially supported by the Slovenian Research Agency (program no. P3-0307).

\section{References}

1. Van Lin EN, van der Vight L, Huizenga H, Kaanders JHAM, Visser AG. Set-up improvement in head and neck radiotherapy using a 3D off-line EPID-based correctioon protocol and a customised head and neck support. Radiother Oncol 2003; 68: 137-48. doi: 10.1016/S0167-8140(03)00134-8

2. Houweling $A C$, van der Meer S, van der Wal E, Terhaard CHJ, Raaijmakers CPJ. Improved immobilization using an individual head support in headand-neck cancer patients. Radiother Oncol 2010; 96: 100-3. doi: 10.1016/j. radonc.2010.04.014 
3. Humphreys M, Guerrero Urbano MT, Mubata C, Miles E, Harrington KJ, Bidmead $\mathrm{M}$, et al. Assessment of a customised immobilisation system for head and neck IMRT using electronic portal imaging. Radiother Oncol 2005; 77: 39-44. doi: 10.1016/j.radonc.2005.06.039

4. McKernan B, Bydder S, Ebert M, Waterhouse D, Joseph D. A simple and inexpensive method to routinely produce customized neck supports for patient immobilization during radiotherapy. J Med Imaging Radiat Oncol 2008; 52: 611-6. doi: 10.1111/j.1440-1673.2008.02024

5. De Boer HC, Heijmen BJ. eNAL: an extenstion of the NAL setup correction protocol for effective use of weekly follow-up measurements. Int J Radiat Oncol Biol Phys 2007; 67: 1586-95. doi: 10.1016/j.jijobp.2006.11.050

6. Howlin C, O'Shea E, Dunne M, Mullaney L, McGarry M, Clayton-Lea A, et al. A randomized controlled trial comparing customized versus standard headrests for head and neck radiotherapy immobilization in terms of set-up errors, patient comfort and staff satisfaction. Radiography 2015; 21: 74-83. doi: 10.1016/j.radi.2014.07.009

7. $\mathrm{R}$ Core Team. R: a language and environment for statistical computing Vienna: R Foundation for Statistical Computing; 2018.

8. Faul F, Erdfelder E, Lang AG, Buchner A. G*Power 3: a flexible statistical power analysis program for the social, behavioral, and biomedical sciences. Behav Res Methods 2007; 39: 175-91. doi: 10.3758/bf03193146

9. Van Herk M. Errors and margins in radiotherapy. Semin Radiat Oncol 2004 14: 52-64. doi: 10.1053/j.semradonc.2003.10.003

10. International Atomic Energy Agency. Introduction of image guided radiotherapy into clinical practice. In: IAEA Human Health Reports No. 16. Vienna: International Atomic Energy Agency; 2019. 